\title{
Examination of the impact of using generic nutritional composition data, on estimation of nutrient intake at both meal and daily food intake levels
}

\author{
C. Woolhead ${ }^{1}$, M. Gibney ${ }^{1}$, M. Marianne ${ }^{1}$, L. Brennan ${ }^{1}$ and E. R. Gibney ${ }^{1}$ \\ ${ }^{1}$ Institute of Food and Health, University College Dublin, Ireland
}

Currently, dietary data, using food diaries or recalls, is collected and analysed at an individual food item level, seeking specifics such as brand, food weight and time of consumption, which can be time-consuming and cumbersome. It remains to be seen whether such detail is required and how generic information application impacts on nutrient intake.

Dietary data, based on 4-day semi-weighed food diaries from the Irish National Adult Nutrition Survey (NANS) was used for this analysis. The data contained 133,068 food occasions and 2552 individually-coded food items. Each food item had details including meal code, study day and food-weight amongst others. For the purpose of this analysis "Day 1" and meal-type "Breakfast" was selected, consisting of 683 food items. These were coded into 21 food groups e.g. "Breads", "Milks". Dietary intake modelling was performed by substituting generic nutritional compositions of specific foods/food groups and the impact of altered compositions was assessed at meal and daily mean intake levels. These generic nutritional compositions were tailored specifically to the weight of the food consumed at each occasion. Food groups examined included "Milk", "Fruit", "Bread" and "Cereal", the latter of which was subsequently split into 3 groups: "Porridge-like", "Muesli" and "Other".

Dietary intake modelling demonstrated no significant differences in mean nutrient intake when substituting "Breads" or "Fruits" with generic compositions at both mean daily and meal intake levels. Using generic compositional data within the food group "Milks", there were no significant differences for the majority of nutrients, except phosphorus $(p \leqslant 0.001)$ and saturated fat $(p \leqslant 0.05)$. "Cereals" (split), at a daily level, produced no significant differences for most nutrients, other than calcium $(p \leqslant 0.001)$ and NSP $(p \leqslant 0.05)$. However, for cereals, many significant differences were found at a meal level $(p \leqslant 0.001)$, including energy, carbohydrate, sugar, calcium, magnesium, phosphorus and dietary fibre; starch, total fat, mono- and poly-unsaturated-fats $(p \leqslant 0.05)$.

For many food groups at the meal-type "breakfast", a generic nutritional composition may be adequate when examining nutrient intakes in the population, giving promise to the idea of meal assessment in the future.

This work was funded by Food4me (KBBE.2010.2.3-02, Project no.265494), http://www.food4me.org/ 